\title{
man \\ Morphological and Molecular Evidence for Two New Species within Russula Subgenus Brevipes from China
}

\author{
Jie Song ${ }^{1}\left(\mathbb{D}\right.$, Haijiao Li ${ }^{2}$, Shijun Wu ${ }^{1}$, Qianqian Chen ${ }^{1}$, Guang Yang ${ }^{1}$, Jinyun Zhang ${ }^{1}$, Junfeng Liang ${ }^{3}$ \\ and Bin Chen ${ }^{4, *}$
}

check for updates

Citation: Song, J.; Li, H.; Wu, S.; Chen, Q.; Yang, G.; Zhang, J.; Liang, J.; Chen, B. Morphological and Molecular Evidence for Two New Species within Russula Subgenus Brevipes from China. Diversity 2022, 14, 112. https://doi.org/10.3390/ d14020112

Academic Editors: Changlin Zhao and Michael Wink

Received: 26 December 2021

Accepted: 2 February 2022

Published: 5 February 2022

Publisher's Note: MDPI stays neutral with regard to jurisdictional claims in published maps and institutional affiliations.

Copyright: (C) 2022 by the authors. Licensee MDPI, Basel, Switzerland. This article is an open access article distributed under the terms and conditions of the Creative Commons Attribution (CC BY) license (https:// creativecommons.org/licenses/by/ $4.0 /)$.
1 Department of Horticulture and Food, Guangdong Eco-Engineering Polytechnic, Guangzhou 510520, China jsong@caf.ac.cn (J.S.); wushijun0128@163.com (S.W.); jasone19881202@163.com (Q.C.); yangguang200510@126.com (G.Y.); zut2010@126.com (J.Z.)

2 National Institute of Occupational Health and Poison Control, Chinese Center for Disease Control and Prevention, Beijing 100050, China; lihaijiao715@126.com

3 Research Institute of Tropical Forestry, Chinese Academy of Forestry, Guangzhou 510520, China; jfliang2000@163.com

4 Institute of Biological and Medical Engineering, Guangdong Academy of Sciences, Guangzhou 510316, China

* Correspondence: binsanity12@caf.ac.cn

\begin{abstract}
Two new Russula species, R. subbrevipes and R. callainomarginis, from China are described based on morphological and molecular characteristics. Russula subbrevipes has thus far only been found in southwestern China at altitudes of higher than $3400 \mathrm{~m}$ and is characterized by a yellow ochre pileal surface, glabrous or tomentose stipe, fruity odor, subglobose to ellipsoid basidiospores, isolated or partially connected warts and pleurocystidia with a cap appendage. Russula callainomarginis is characterized by a cream to white pileus, light turquoise lamellae margin, spongy stipe, light turquoise zone on the top of the stipe, pungent odor, globose to ellipsoid basidiospores and dominant isolated warts. The phylogenetic tree of Russula was constructed with multi-gene sequences, including the internal transcribed spacer regions (ITS), the ribosomal large subunit (nrLSU), the small subunit of the mitochondrial rRNA gene (mtSSU) and the second largest subunit of RNA polymerase II (RPB2). The results show that both $R$. subbrevipes and R. callainomarginis represent new lineages in Russula subg. Brevipes. Description and illustration of the two new species are presented.
\end{abstract}

Keywords: Russulales; Russulaceae; ectomycorrhizal fungi; phylogeny; taxonomy

\section{Introduction}

Russula Pers. was erected by Persoon [1] and typified by R. emetica (Schaeff.) Pers. It is an important ectomycorrhizal genus worldwide, comprising more than 2000 species [2,3]. Species in the genus play a significant role in forest ecosystems, and many species are harvested for human consumption, especially in China [4,5]. The genus Russula has had a rich taxonomic history during the last two hundred years, and numerous infrageneric classification systems have been proposed [6-9]. Recent molecular phylogenetic studies based on a worldwide representative sampling have indicated eight subgenera within the genus: Russula subg. Glutinosae Buyck and X.H. Wang; R. subg. Archaeae Buyck and Hofst.; R. subg. Compactae (Fr.) Bon; R. subg. Crassotunicatae Buyck and Hofst.; R. subg. Heterophyllidiae Romagnesi; R. subg. Malodorae Buyck and Hofst.; R. subg. Brevipes Buyck and Hofst.; and R. subg. Russula [10,11]. Russula subg. Brevipes Buyck and Hofst was initially recognized as a lineage in the section Plorantinae belonging to $R$. subg. Compactae (Fr.) Bon.

Russula subg. Compactae is characterized by its large-to-small and hard fruiting bodies; dull-colored, white, brown, grey to black pilus; numerous lamellulae alternating with the lamellae; a poorly differentiated pileipellis that does not separate easily from the context; reddening, greying, blackening or browning context; white spore-print [8,12]. It was split into two subtaxa by Bataille in 1908: Lactaroieae (later Plorantinae), which do not blacken, 
and Nigricantinae, which do. Romagnesi [6] followed this system with a minor modification to establish a new classification system, in which the sections Archaeinae Heim: Romagn., Nigricantinae Bataille and Plorantinae Bataille were included in $R$. subg. Compactae.

Recently, phylogenetic analyses were conducted to gain an insight into the genus Russula, and the results showed that sections Archaeinae, Nigricantinae and Plorantinae were well-supported, although they may not be as closely related as previously believed $[4,13,14]$. Buyck et al. [10] firstly studied the morpho-anatomical features of both fruiting bodies, as well as below-ground structures of Russulaceae, and presented a more comprehensive phylogeny based on nrLSU, mtSSU, RPB1, RPB2 and TEF1 sequences. A new classification system was proposed, and the sections Archaeinae, Nigricantinae and Plorantinae were elevated to four different subgenera with significant support: $R$. subg. Archaea, R. subg. Compactae, R. subg. Malodora and R. subg. Brevipes [10,12].

The members in $R$. subg. Brevipes are mostly medium to very large and thick-fleshed, only exceptionally small and thin-fleshed. Cap whitish, often rapidly developing yellowish brown to reddish brown stains. Well-developed annulus never present. Gills regularly unequal. Context turning yellowish to rusty brown, mostly with distinct smell, acrid to strongly acrid. Spore-print whitish to yellow. Spores with inamyloid or amyloid suprahilar spot. Primordial hyphae absent. Gloeocystidia mucronate to obtuserounded in all parts of the fruiting body. Hyphal extremities of cap surface inflated or not [10].

This subgenus has a cosmopolitan distribution. In Europe, the group is represented by the well-known $R$. chloroides (Krombh.) Bres. and R. delica Fr. In Asia, R. subg. Brevipes is frequently reported from the Himalayas and Kashmir, and Li et al. reported three new species (R. leucocarpa G.J. Li and C.Y. Deng; R. byssina G.J. Li and C.Y. Deng; R. cremicolor G.J. Li and C.Y. Deng) from Guizhou Province of China in 2020, based on ITS sequences [15]. However, the taxonomy of this group is exceptionally challenging due to nomenclatoric mess and dubious taxa within $R$. delica and $R$. chloroides $[3,10]$.

This paper is part of an ongoing project in which taxonomic and phylogenetic studies focusing on Russula in China are being carried out to clarify the species diversity. Some new species have been described in recent years [16-19]. As a continuation of these surveys, two Russula species were found to be undescribed. Phylogenetic analyses based on ITS, nrLSU, $\mathrm{mtSSU}$ and RPB2 regions were carried out to verify their affinity within the $R$. subg. Brevipes.

\section{Materials and Methods}

\subsection{Morphological Studies}

Collections were obtained from southwestern China during 2012-2015. Photographs and macro-morphological descriptions were based on fresh, mature fruitbodies, and specimens were then dried in an oven at $40{ }^{\circ} \mathrm{C}$ until completely desiccated. The studied specimens were deposited in the herbarium of Research Institute of Tropical Forestry, Chinese Academy of Forestry (RITF), the Herbarium of Cryptogams, Kunming Institute of Botany, Chinese Academy of Sciences (HKAS) and the personal herbarium of Haijiao Li (LI). Terminology for descriptive terms follows Vellinga [20]. The description templates and terminology of the micromorphological characters were taken from Adamčík, et al. [3]. Color names and codes follow Kornerup and Wanscher [21].

Microscopic examinations followed Adamčík, et al. [3]. Tissues of specimens were first immersed in $5 \%$ potassium hydroxide $(\mathrm{KOH})$ and then stained with $1 \%$ aqueous Congo red solution for microscopic observation with an Axio Imager 2 upright microscope (Carl Zeiss Microscopy, GmbH, Germany) under a $100 \times$ oil immersion objective lens. Observations and measurements of the basidiospores and ornamentation were made in Melzer's reagent. All tissues were also examined in Cresyl blue to verify presence of ortho- or metachromatic reactions as explained in Buyck [22]. Sulphovanillin (SV) solution was used to test for reactions of cystidia. Scanning electron microscope (SEM) photos were captured with a JEOL JSM-6510 microscope (Tokyo, Japan). The abbreviation ( $\mathrm{n} / \mathrm{m} / \mathrm{p})$ indicates $\mathrm{n}$ basidiospores measured from $\mathrm{m}$ fruit bodies of $\mathrm{p}$ specimens. Basidiospore measurements are presented as (Min-)AV-SD-AV-AV+SD(-Max), where Min is the minimum value, Max is the maxi- 
mum value, $\mathrm{AV}$ is the average value, $\mathrm{SD}$ is the standard deviation, and $\mathrm{Q}$ represents the length/width ratio of the basidiospores.

\subsection{Molecular Study and Phylogenetic Analysis}

Genomic DNA was extracted from dried mushrooms with the CTAB protocol [23]. The DNA was amplified with the primers: ITS4 and ITS5 for ITS [24]; LR0R and LR7 for nrLSU [25]; RPB2 using the primers bRPB2-6f and fRPB2-7cr [26,27]; the mitochondrial small subunit (mtSSU) with primers MS1 and MS2 [24].

Amplifications were performed in a $50 \mu \mathrm{L}$ reaction volume containing $5 \mu \mathrm{L}$ of $10 \times \mathrm{PCR}$ reaction buffer, $5 \mu \mathrm{L}$ dNTP mix $(0.2 \mathrm{mmol}), 2 \mu \mathrm{L}$ each of primers $(5 \mu \mathrm{mol})$ and $1.5 \mathrm{U}$ of Taq DNA polymerase. The final volume was adjusted to $50 \mu \mathrm{L}$ with sterile distilled $\mathrm{H}_{2} \mathrm{O}$ [28]. The PCR procedure for ITS, $\mathrm{mtSSU}$ and RPB2 was as follows: initial denaturation at $95^{\circ} \mathrm{C}$ for $3 \mathrm{~min}$, followed by 35 cycles at $94{ }^{\circ} \mathrm{C}$ for $40 \mathrm{~s}, 48{ }^{\circ} \mathrm{C}$ for $45 \mathrm{~s}, 72{ }^{\circ} \mathrm{C}$ for $1 \mathrm{~min}$ and a final extension of $72{ }^{\circ} \mathrm{C}$ for $10 \mathrm{~min}$. The PCR procedure for nrLSU was as follows: initial denaturation at $94{ }^{\circ} \mathrm{C}$ for $1 \mathrm{~min}$, followed by 35 cycles at $94{ }^{\circ} \mathrm{C}$ for $30 \mathrm{~s}, 50{ }^{\circ} \mathrm{C}$ for $1 \mathrm{~min}$, $72{ }^{\circ} \mathrm{C}$ for $1.5 \mathrm{~min}$ and a final extension of $72{ }^{\circ} \mathrm{C}$ for $10 \mathrm{~min}$. The PCR products were visualized by agarose gel electrophoresis and stored at $-20{ }^{\circ} \mathrm{C}$ after visualization. The PCR products were purified by using TaKaRa MiniBEST Agarose Gel DNA Extraction Kit according to the operation manual. DNA sequencing was performed with an ABI 3730 DNA analyzer and an ABI BigDye 3.1 terminator cycle sequencing kit (Shanghai Sangon Biological Engineering Technology and Services Co. Ltd., Shanghai, China). The basic authenticity and reliability of newly generated sequences were established based on Nilsson et al. [29]. All newly generated sequences were submitted to GenBank and are listed in Table 1.

Table 1. A list of species, specimens, and GenBank accession number of sequences used in this study.

\begin{tabular}{|c|c|c|c|c|c|c|}
\hline \multirow{2}{*}{ Species } & \multirow{2}{*}{ Collection No. } & \multirow{2}{*}{ Location } & \multicolumn{4}{|c|}{ GenBank Accession No. } \\
\hline & & & ITS & LSU & $R P B 2$ & mtSSU \\
\hline Lactifluus piperatus & $\begin{array}{l}\text { M. Lecomte:2001 } \\
081955\end{array}$ & France & KF220121 & KF220214 & KF220287 & NC_038056 \\
\hline Russula acrifolia & $r-05065$ & USA & JF834363 & JF834510 & JF834460 & - \\
\hline R. acrifolia & BB 08.662 & Italy & - & KU237535 & KU237821 & KU237381 \\
\hline R. acrifolia & RITF3122 & China & MH911600 ${ }^{\mathrm{a}}$ & MH911611 $^{\mathrm{a}}$ & MH911626 ${ }^{\mathrm{a}}$ & - \\
\hline R. adusta & 5226 & Italy & JF908669 & - & - & - \\
\hline R. adusta & BB 06.562 & Canada & - & KU237476 & KU237762 & KU237320 \\
\hline R. albonigra & r-04105 & USA & JF834355 & JF834503 & JF834452 & - \\
\hline R. albonigra & BB 07.291 & Slovakia & & KU237536 & KU237822 & KU237382 \\
\hline R. amara & FH12213 & Germany & КТ933998 & KT933859 & КТ933930 & - \\
\hline R. amoenolens & BPL232 & USA & KT933954 & КТ933813 & КТ933884 & - \\
\hline R. archaeosuberis & BB 12.085 & Italy & KY800355 & KU237593 & KU237878 & KU237441 \\
\hline$R$. aff. areolata & BB 06.090 & Madagascar & - & KU237471 & KU237757 & KU237315 \\
\hline R. australis & JAC10732 & New Zealand & MW683746 & MW683616 & & \\
\hline R. azurea & BB 08.668 & Italy & JN944002 & KU237529 & KU237815 & KU237375 \\
\hline R. blennia sp. ined. & BB 08.066 & Madagascar & - & KU237556 & KU237842 & KU237404 \\
\hline R. brevipes & BB 06.508 & Mexico & - & KU237479 & KU237765 & KU237323 \\
\hline R. cf. brevipes & BB 06.441 & Mexico & - & KU237483 & KU237769 & KU237327 \\
\hline R. brevipes var. acrior & JMP0058 & USA & EU819422 & & & \\
\hline R. callainomarginis & RITF2639 & China & $\mathrm{MH} 286463^{\mathrm{a}}$ & $\mathrm{MH} 286468^{\mathrm{a}}$ & MH911624 ${ }^{\mathrm{a}}$ & MH911616 ${ }^{\mathrm{a}}$ \\
\hline R. callainomarginis & Li160714-03 & China & MH911604 ${ }^{\mathrm{a}}$ & - & - & - \\
\hline R. callainomarginis & Li150731-09 & China & MH911605 & - & - & - \\
\hline R. callainomarginis & Li160910-20 & China & MH911606 & - & - & - \\
\hline R. callainomarginis & Li160714-04 & China & MH911607 ${ }^{\mathrm{a}}$ & - & - & - \\
\hline
\end{tabular}


Table 1. Cont.

\begin{tabular}{|c|c|c|c|c|c|c|}
\hline \multirow{2}{*}{ Species } & \multirow{2}{*}{ Collection No. } & \multirow{2}{*}{ Location } & \multicolumn{4}{|c|}{ GenBank Accession No. } \\
\hline & & & ITS & LSU & $R P B 2$ & mtSSU \\
\hline R. camarophylla & PAM01081108 & China & DQ421982 & DQ421982 & DQ421938 & - \\
\hline R. cf. camarophylla & MPG11-7-09 & Spain & - & KU237579 & KU237865 & KU237427 \\
\hline R. capillaris sp. ined. & BB 08.172 & Madagascar & - & KU237553 & KU237839 & KU237399 \\
\hline$R$. aff. cellulata & BB 06.045 & Madagascar & - & KU237454 & KU237740 & KU237298 \\
\hline R. chloroides & UBCF20353 & Canada & КC581331 & KC581331 & - & - \\
\hline$R$. aff. chloroides & FH12273 & Belgium & KT934015 & KT933876 & КT933947 & \\
\hline R. compacta & BPL227 & USA & KT933952 & KT933810 & KT933881 & - \\
\hline R. compacta & BB 06.295 & USA & - & KU237480 & KU237766 & KU237324 \\
\hline R. cortinarioides & BB 07.103 & USA & КР033480 & KP033491 & KP033502 & KU237402 \\
\hline R. cuprea & FH12250 & Slovakia & KT934010 & KT933871 & KT933942 & - \\
\hline R. decipiens & SAV F-1022 & Slovakia & KY582683 & - & KY616679 & KY471572 \\
\hline R. decolorans & FH12196 & Slovakia & KT933992 & KT933853 & KT933924 & - \\
\hline R. delica & FH12272 & Belgium & KF432955 & KR364224 & KR364340 & - \\
\hline R. aff. delica & BB 12.086 & Italy & - & KU237594 & KU237879 & KU237442 \\
\hline R. cf. delica & SA07.210 & Slovakia & - & KU237600 & KU237885 & KU237449 \\
\hline R. delicinae & BB 06.476 & Mexico & - & KU237484 & KU237770 & KU237328 \\
\hline R. densifolia & BB 07.344 & Slovakia & - & KU237502 & KU237788 & KU237347 \\
\hline R. dissimulans & OSA-MY-1727 & Japan & AB291731 & AB154717 & - & - \\
\hline R. earlei & BPL245 & USA & KT933961 & KT933820 & КТ933891 & - \\
\hline R. aff. earlei & MT s.n. & Costa Rica & - & KU237598 & KU237883 & KU237446 \\
\hline R. eccentrica & BB 07.044 & USA & КР033479 & КР033490 & КР033501 & KU237353 \\
\hline R. cf. eccentrica & BB 07.132 & USA & КР033478 & КР033489 & КР033500 & KU237341 \\
\hline R. edulis & BB 08.167 & Madagascar & - & KU237564 & KU237850 & KU237412 \\
\hline R. fellea & FH12185 & Slovakia & КТ933989 & KT933850 & KT933921 & - \\
\hline R. fistulosinae sp. ined. & BB 08.105 & Madagascar & - & KU237527 & KU237813 & KU237373 \\
\hline R. fragilis & FH12197 & France & КТ933993 & KT933854 & KT933925 & - \\
\hline R. globispora & $\begin{array}{c}\text { GENT:FH-2007- } \\
\text { BT111 }\end{array}$ & Germany & KU928144 & - & KY616671 & KY471564 \\
\hline R. gossypina & BB 06.002 & Madagascar & - & KU237450 & KU237736 & KU237293 \\
\hline R. griseobrunnea & PDD:81525 & $\begin{array}{l}\text { New } \\
\text { Zealand }\end{array}$ & GU222265 & - & - & - \\
\hline R. aff. griseobrunnea & BB 09.344 & $\begin{array}{c}\text { New } \\
\text { Caledonia }\end{array}$ & - & KU237592 & KU237877 & KU237440 \\
\hline R. hatsikiana sp. ined. & BB 08.178 & Madagascar & - & KU237557 & KU237843 & KU237405 \\
\hline R. herrerae & BB 06.532 & Mexico & - & KU237486 & KU237772 & KU237330 \\
\hline R. inornata sp. ined. & BB 08.194 & Madagascar & - & KU237558 & KU237844 & KU237406 \\
\hline R. integra & FH12172 & Slovakia & КT933984 & KT933845 & КT933916 & - \\
\hline R. laeta & SAV F-3949 & Slovakia & KY582708 & - & KY616709 & KY471600 \\
\hline R. laevis & JR4016 & Finland & MN130091 & MN130128 & MN380529 & MN161180 \\
\hline R. laricina & $575 / 08.681$ & Italy & JN944008 & JN940593 & KU237846 & - \\
\hline R. lateriticola & BB 06.031 & Madagascar & KP033476 & KP033487 & KP033498 & KU237297 \\
\hline R. lepida & HJB9990 & Belgium & DQ422013 & DQ422013 & DQ421954 & KY471624 \\
\hline R. cf. liberiensis & BB 06.184 & Madagascar & - & KU237474 & KU237760 & KU237318 \\
\hline R. lilacea & BB 07.213 & Slovakia & JN944005 & KU237498 & KU237784 & KU237343 \\
\hline R. luteotacta & FH12187 & Slovakia & КT933991 & KT933852 & KT933923 & - \\
\hline R. marangania & MEL2293694 & Australia & EU019930 & EU019930 & & \\
\hline R. mariae & SFC20120922-08 & South Korea & KF361778 & KF361828 & KF361728 & - \\
\hline R. melliolens & SAV F-4201 & Slovakia & KY582719 & - & KY616712 & KY471611 \\
\hline R. minutula & BB 08.636 & Italy & - & KU237531 & KU237817 & KU237377 \\
\hline R. mustelina & FH12226 & Germany & КT934005 & KT933866 & KT933937 & - \\
\hline R. nauseosa & FH12173 & Germany & KT933985 & KT933846 & KT933917 & - \\
\hline R. nigricans & UE20.09.2004-07 & Sweden & DQ422010 & DQ422010 & - & - \\
\hline R. nigricans & BB 07.342 & Slovakia & - & KU237495 & KU237781 & KU237339 \\
\hline R. ochroleuca & FH12211 & Germany & КT933996 & KT933857 & КT933928 & - \\
\hline
\end{tabular}


Table 1. Cont.

\begin{tabular}{|c|c|c|c|c|c|c|}
\hline \multirow{2}{*}{ Species } & \multirow{2}{*}{ Collection No. } & \multirow{2}{*}{ Location } & \multicolumn{4}{|c|}{ GenBank Accession No. } \\
\hline & & & ITS & LSU & $R P B 2$ & mtSSU \\
\hline R. odorata & BB 07.186 & Slovakia & JN944010 & KU237518 & KU237804 & KU237364 \\
\hline R. pallidospora & JV02-218 & Sweden & DQ422032 & DQ422032 & - & - \\
\hline R. aff. pallidospora & MPG13-6-08 & Spain & - & KU237580 & KU237866 & KU237428 \\
\hline R. pectinatoides & BPL276 & USA & КТ933975 & KT933836 & КТ933907 & - \\
\hline R. pelargonia & $r-04023$ & USA & JF834348 & JF834496 & JF834445 & - \\
\hline R. persicina & UE21.09.2003-01 & Sweden & DQ422019 & DQ422019 & DQ421960 & - \\
\hline R. polyphylla & BB 07.134 & USA & КР033486 & КР033497 & КР033508 & KU237448 \\
\hline R. polyphylla & BB 07.023 & USA & KP033481 & KP033492 & KP033503 & KU237403 \\
\hline R. polyphyllinae sp. ined. & BB 09.215 & $\begin{array}{c}\text { New } \\
\text { Caledonia }\end{array}$ & - & KU237590 & KU237875 & KU237438 \\
\hline R. pseudoaurantiophylla & BB 09.219 & $\begin{array}{c}\text { New } \\
\text { Caledonia }\end{array}$ & - & KU237591 & KU237876 & KU237439 \\
\hline R. pumicoidea & Trappe14771 & Australia & EU019931 & EU019931 & & \\
\hline R. raoultii & FH12222 & Germany & KT934002 & KT933863 & KT933934 & - \\
\hline R. romellii & FH12177 & Germany & KT933987 & KT933848 & КТ933919 & - \\
\hline R. sesenagula & BB 08.117 & Madagascar & - & KU237526 & KU237812 & KU237372 \\
\hline R. sinuata & H4755 & Australia & EU019943 & & & \\
\hline R. solaris & BB 07.282 & Slovakia & JN944007 & JN940606 & KU237835 & KU237395 \\
\hline R. subbrevipes & RITF3136 & China & $\mathrm{MH} 286460^{\mathrm{a}}$ & MH286465 & MH911625 & $\mathrm{MH} 911617^{\mathrm{a}}$ \\
\hline R. subbrevipes & RITF2946 & China & $\mathrm{MH} 286462^{\mathrm{a}}$ & $\mathrm{MH} 286467^{\mathrm{a}}$ & - & MH911618 \\
\hline R. subbrevipes & RITF3002 & China & $\mathrm{MH}_{286461}^{\mathrm{a}}$ & $\mathrm{MH} 286466^{\mathrm{a}}$ & - & $\mathrm{MH} 11619^{\mathrm{a}}$ \\
\hline R. cf. subfistulosa & BB 08.176 & Madagascar & - & KU237542 & KU237828 & KU237388 \\
\hline R. subnigricans & RITF2657 & China & MH911602 ${ }^{\mathrm{a}}$ & MH911612 ${ }^{\mathrm{a}}$ & - & MH911620 ${ }^{\mathrm{a}}$ \\
\hline R. subnigricans & Li160821-05 & China & MH911603 ${ }^{\mathrm{a}}$ & - & - & - \\
\hline R. subnigricans & YM-64 & China & MH911601 ${ }^{\mathrm{a}}$ & - & - & - \\
\hline R. vesca & BPL284 & USA & KT933978 & KT933839 & KT933910 & - \\
\hline R. vesicatoria & BB 07.034 & USA & - & KU237599 & KU237884 & - \\
\hline R. violeipes & SFC20121010-06 & South Korea & KF361808 & KF361858 & KF361758 & - \\
\hline R. zvarae & FH12175 & Germany & KT933986 & KT933847 & КТ933918 & - \\
\hline
\end{tabular}

a New sequences for this study.

Sequences were aligned in MAFFT 7 ([30]; http:/ / mafft.cbrc.jp/alignment/server/, accessed on 22 January 2022) using the "G-INS-I" strategy and manually adjusted in BioEdit [31]. One thousand partition homogeneity test (PHT) replicates of ITS, nrLSU, $\mathrm{mtSSU}$ and RPB2 sequences were tested by PAUP* $\mathrm{v} 4.0 \mathrm{~b} 10$ [32] to determine whether the partitions were homogeneous. The PHT results indicated all the DNA sequences display a congruent phylogenetic signal ( $p$-value $=0.03$ ). This means that the genes' sequence dataset did not show any conflicts in tree topology for the reciprocal bootstrap trees, which allowed us to combine them. Sequences of species and outgroup Lactifluus piperatus (L.) Kuntze were retrieved from GenBank (NCBI) and combined with the new sequences to construct a concatenated ITS $+\mathrm{nrLSU}+\mathrm{mtSSU}+\mathrm{RPB} 2$ dataset. Sequence alignment was deposited at TreeBase (submission ID 23830).

The best-fit model of nucleotide evolution to the datasets was selected with AIC (Akaike Information Criterion) using MrModeltest 2.3 [33,34]. Best model for the combined ITS+nrLSU+mtSSU+RPB2 sequence dataset estimated was GTR. Bayesian inference (BI) and maximum likelihood (ML) analyses were applied to the dataset with four partitions (ITS, nrLSU, mtSSU and RPB2).

BI analysis was performed using MrBayes on XSEDE (3.2.6) on Abe through the Cipres Science Gateway (www.phylo.org, accessed on 27 January 2022) with 2 independent runs, each one beginning from random trees with 4 simultaneous independent chains, performing 2 million replicates, sampling one tree every 1000th generation. The first $25 \%$ of the sampled trees were discarded as burn-in, and the remaining ones were used to reconstruct a majority rule consensus and calculate Bayesian posterior probabilities (BPP) of the clades. 
ML analysis searches were conducted with RAxML-HPC2 on XSEDE (8.2.10) on Abe through the Cipres Science Gateway (www.phylo.org, accessed on 27 January 2022 involving $100 \mathrm{ML}$ searches under the GTRGAMMA model, with all model parameters estimated by the program. Only the maximum-likelihood best tree from all searches was kept. In addition, 100 rapid bootstrap replicates were run with the GTRCAT model to assess the reliability of the nodes.

Branches that received bootstrap support for maximum likelihood (BS) and Bayesian posterior probabilities (BPP) greater than or equal to $75 \%$ (BS) and 0.95 (BPP), respectively, were considered as significantly supported.

\section{Results}

\subsection{Molecular Phylogeny}

The combined dataset included sequences from 101 fungal specimens or isolates representing 82 taxa. The dataset had an aligned length of 3108 characters including gaps, of which 2065 characters were constant, 247 were variable and parsimony-uninformative and 796 were parsimony-informative. Bayesian analysis and ML analysis resulted in a similar topology, with an average standard deviation of split frequencies of 0.008966 (BI). The ML topology is shown in Figure 1.

The phylogenetic analyses revealed that the subgenera proposed by Buyck et al. (2018) were well-supported with significant BI and ML values: Russula subg. Malodora $(\mathrm{BS}=97 \%$, $\mathrm{BPP}=1.00)$; Russula subg. Brevipes (BS =97\%, BPP =1.00); Russula subg. Compactae $(\mathrm{BS}=98 \%, \mathrm{BPP}=1.00)$ and Russula subg. Archaea $(\mathrm{BS}=100 \%, \mathrm{BPP}=1.00)$. Our collections from Southern China formed two new lineages (bold names in Figure 1) with significant support $(\mathrm{BS}=100 \%, \mathrm{BPP}=1.00 ; \mathrm{BS}=100 \%, \mathrm{BPP}=1.00$, respectively $)$ and fell into the Russula subg. Brevipes. They were considered as distinct phylogenetic species.

\subsection{Taxonomy}

Russula subbrevipes J.F. Liang and J. Song, sp. nov. (Figures 2-4)

MycoBank: MB 829447 (https: / / www.mycobank.org/page/Name\%20details\%20pag e/571620).

Diagnosis-Differs from other Russula species in this group by yellow ochre pileus, tomentose stipe, fruity odor, cylindrical or clavate pleurocystidia with a cap appendage, subglobose to ellipsoid basidiospores ((7.0-)7.8-8.4-9.0(-9.7) × (6.4-)6.9-7.4-7.9(-8.4) $\mu \mathrm{m})$, amyloid ornamentation with isolated or partial connected warts.

Etymology-Subbrevipes (Lat.): refers to the morphological similarity to $R$. brevipes Peck.

Holotype-CHINA. Yunnan Province, Diqing Tibetan Autonomous Prefecture, Shangrila, Pudacuo National Park, $27^{\circ} 51^{\prime} 17^{\prime \prime}$ N, $99^{\circ} 57^{\prime} 8^{\prime \prime}$ E, elev. $3400 \mathrm{~m}$, on ground of Quercus, Betula and Pinus, 25 August 2014, Zhao 2265 (RITF3136).

Description-basidiomata medium-sized to large. Pileus $90-140 \mathrm{~mm}$ in diam., first hemispherical, expanding to plano-concave with a depressed center, then broadly infundibuliform when mature; margin incurved when juvenile, not becoming straight, smooth, without striate, sometimes dehiscent with age; surface yellow ochre (5C7) when fresh, becoming pale yellow (1A3) to cinnamon (6D6) when dry, slightly viscid when moist, glabrous, smooth, without striate; suprapellis unpeelable; context hard, up to $5 \mathrm{~mm}$ thick towards center, white (1A1) when fresh, unchanging when bruising. Lamellae subdecurrent, close to crowded with 1-3 series lamellulae, no forking near the stipe, white when fresh, becoming brownish-orange (5C3) to brown (5E5) when dry, unchanging when bruising. Stipe 40-50 × 10-25 mm, cylindrical, solid, cream (4A3) to white (1A1), becoming pale yellow (1A3) when dry, unchanging when bruising, glabrous or tomentose on the upper half part. Odor fruity. Taste mild. Spore-print whitish. 


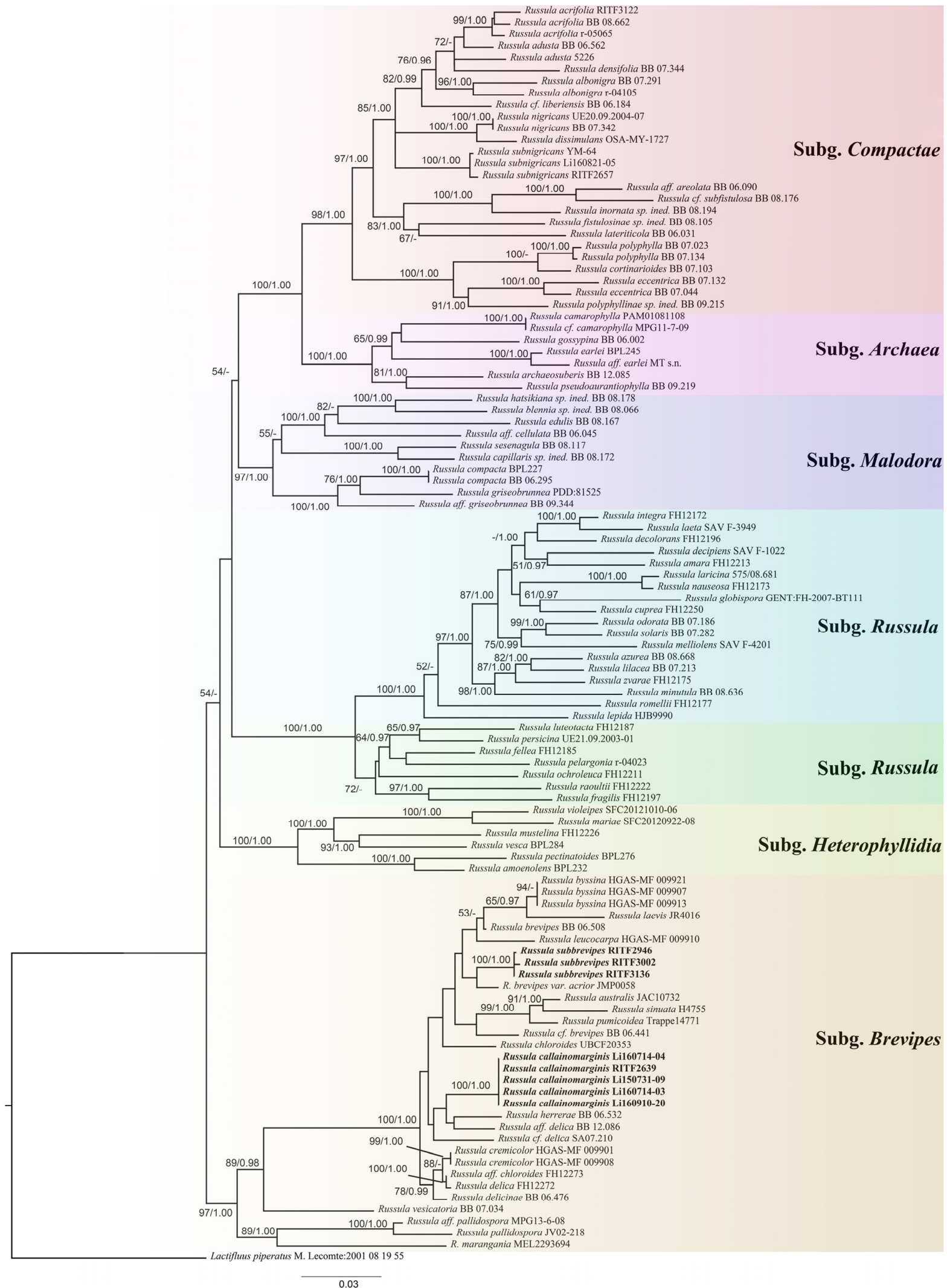

Figure 1. Phylogenetic consensus tree inferred from the maximum likelihood (ML) analysis based on ITS+nrLSU+mtSSU+RPB2 sequence data. Branches are labelled with maximum-likelihood bootstrap proportions (BS) higher than $50 \%$ and Bayesian posterior probabilities (BPP) more than 0.95: bold names $=$ new species. 


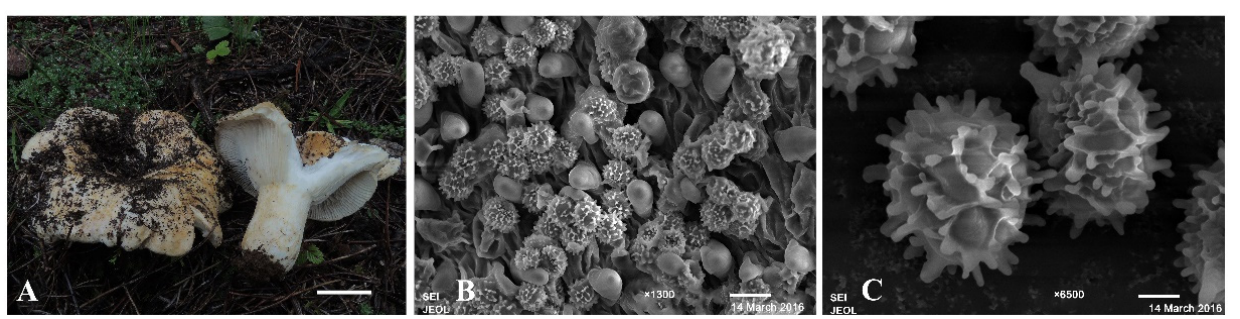

Figure 2. Basidiomata and microscopic structures of Russula subbrevipes (drawn from the holotype). (A) Basidiomata. (B,C) Basidiospores (SEM). Scale bars: $(\mathbf{A})=3 \mathrm{~cm} ;(\mathbf{B})=10 \mu \mathrm{m} ;(\mathbf{C})=2 \mu \mathrm{m}$.
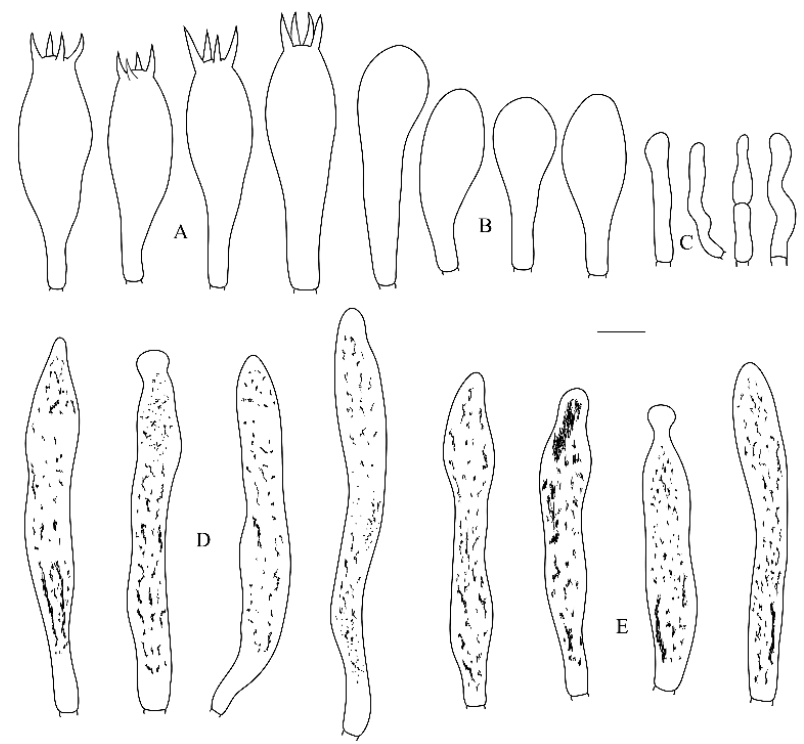

Figure 3. Russula subbrevipes (RITF3136). (A) Basidia. (B) Basidiola. (C) Marginal cells. (D) Hymenial cystidia on lamellae sides. (E) Hymenial cystidia on lamellae edges. Scale bar: $(\mathbf{A}-\mathbf{E})=10 \mu \mathrm{m}$.
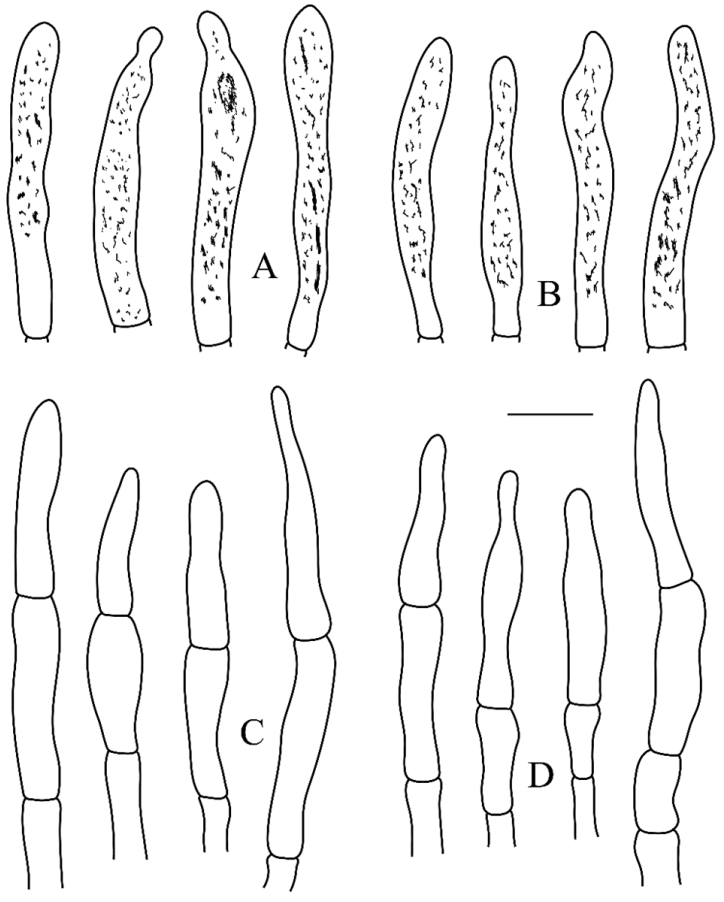

Figure 4. Russula subbrevipes (RITF3136). (A) Pileocystidia near the pileus margin. (B) Pileocystidia near the pileus center. (C) Hyphal terminations near the pileus margin. (D) Hyphal terminations near the pileus center. Scale bar: $(\mathbf{A}-\mathbf{D})=10 \mu \mathrm{m}$. 
Basidiospores (7.0-)7.8-8.4-9.0(-9.7) × (6.4-)6.9-7.4-7.9(-8.4) $\mu \mathrm{m}, \mathrm{Q}=(1.01-) 1.08-$ 1.14-1.21(-1.31), subglobose to ellipsoid; ornamentation amyloid; warts bluntly conical to subcylindrical, up to $1.4 \mu \mathrm{m}$ high, isolated or more commonly with light to heavy connectives forming a partial reticulum; suprahilar plage distinct, weakly amyloid. Basidia (37.0-)40.0-44.5-49.1(-52.3) × (11.2-)6.9-7.4-7.9(-15.9) $\mu \mathrm{m}$, mostly 4-spored, mainly clavate; basidiola clavate or ellipsoid, ca. 9-14 $\mu \mathrm{m}$ wide. Hymenial cystidia on lamellae sides moderately numerous, (59.5-)64.0-72.5-81.0(-91.0) × (8.5-)9.0-9.7-10.3(-10.5) $\mu \mathrm{m}$, mainly clavate or fusiform, apically often obtuse, sometimes with a cap appendage, thin-walled; contents heteromorphous or granulose, turning dark grey in SV. Hymenial cystidia on lamellae edges similar to on lamellae sides in shape and contents, but often shorter, (50.6-)54.0-61.168.3(-74.0) × (7.0-)7.9-9.3-10.6(-12.6) $\mu \mathrm{m}$. Marginal cells (15.0-)17.9-20.8-23.8(-25.8) $\times$ (4.0-)4.4-5.1-5.8(-6.7) $\mu \mathrm{m}$, subcylindrical, fusiform or lageniform, often flexuous. Pileipellis orthochromatic in Cresyl blue, sharply delimited from the underlying spherocytes of the context, ca. 150-285 $\mu \mathrm{m}$ deep; two layered with subpellis ca. 60-85 $\mu \mathrm{m}$ deep, horizontally oriented, intricate, less gelatinized, dense hyphae, 3-5.5 $\mu$ m wide, and ca. 90-200 $\mu \mathrm{m}$ deep suprapellis of strongly gelatinized, repent, loose arranged hyphae, $3-5.5 \mu \mathrm{m}$ wide. Hyphal terminations near the pileus margin rarely branched, sometimes flexuous, thin-walled, terminal cells 19.0-23.8-29.0(-33.2) × (3.6-)4.0-4.9-5.7(-6.3) $\mu \mathrm{m}$, mainly attenuated or narrowly lageniform, occasionally subcylindrical, apically constricted or obtuse; subterminal cells frequently wider, ca. 4-7 $\mu \mathrm{m}$ wide, typically unbranched. Hyphal terminations near the pileus center similar to those near the pileus margin, (20.3-)21.8-25.8-29.8(-33.2) $\times$ (3.7-)4.2-4.8-5.4(-5.7) $\mu \mathrm{m}$, mainly lageniform, occasionally subcylindrical; subterminal cells often shorter, ca. 3-6 $\mu \mathrm{m}$ wide, typically unbranched. Pileocystidia near the pileus margin always one-celled, scattered, (31.3-)33.9-39.5-45.1(-47.5) × (4.5-)4.9-5.7-6.6(-7.0) $\mu \mathrm{m}$, mainly clavate, occasionally fusiform, apically typically obtuse, sometimes with a globose appendage, thin-walled; contents heteromorphous, blackish in SV. Pileocystidia near the pileus center similar to pileus margin in shape, size and contents, always onecelled, scattered, 32.0-37.6-43.0(-46.5) × 4.6-5.7-6.9(-8.0) $\mu \mathrm{m}$. Clamp connections absent in all tissues.

Habitat and distribution-single or scattered in forests dominated by Quercus sp., Betula sp. and Pinus sp. at altitudes greater than $3400 \mathrm{~m}$ in south-western China.

Additional specimens (paratypes) examined-CHINA. Yunnan Province: Diqing Tibetan Autonomous Prefecture, Shangri-la, Pudacuo National Park, 25 August 2014, T25446 (RITF2946) and T25575 (RITF3002).

Russula callainomarginis J.F. Liang and J. Song, sp. nov. (Figures 5-7)

MycoBank: MB 829448 (https:/ / www.mycobank.org/page/Name\%20details\%20pag e/571632).

Diagnosis-differs from other Russula species in this group by its cream to white pileus, adnate and folded lamellae with 1-2 series lamellulae, light turquoise lamellae margin, spongy stipe, pungent odor, globose to ellipsoid basidiospores $((6.4-) 6.8-7.4-8.0(-8.5) \times$ (5.2-)6.0-6.5-7.1(-7.5) $\mu \mathrm{m})$, amyloid ornamentation and isolated warts.

Etymology-Callainomarginis (Lat.): referring to the light turquoise lamellae margin.

Holotype-CHINA. Hubei Province, Shennongjia Forestry District, Longjiangping, $31^{\circ} 26^{\prime} 36^{\prime \prime} \mathrm{N}, 110^{\circ} 29^{\prime} 18^{\prime \prime}$ E, elev. $1850 \mathrm{~m}, 10$ August 2015, LYK 91 (RITF2639).

Description-basidiomata medium-sized. Pileus $60-80 \mathrm{~mm}$ in diam., first hemispherical, then developing convex to umbilicate, centrally depressed; margin usually remaining somewhat decurved and inrolled even when dry; surface cream to white (1A1) when fresh, becoming wax yellow (3A5) to greyish-orange (5B6) upon drying, nonviscid when wet, glabrous, smooth, not striated, never cracked; suprapellis unpeelable; context hard, up to $7 \mathrm{~mm}$ thick towards center, white (1A1) when fresh, color unchanged when bruised. Lamellae adnate, usually folded, close with 1-2 series lamellulae, no forking seen near the stipe, white when fresh, becoming light brown spots and stains when bruised, becoming silver-white (2B2) to sienna (6D7) when dry, lamellae margin light turquoise (24A5) when young, becoming cream (4A3). Stipe up to $40 \mathrm{~mm}$ long, $16 \mathrm{~mm}$ wide, cylindrical with 
slightly tapered base, white (1A1) when fresh with light turquoise (24A5) zone on the apex, becoming pale yellow (1A3) when dry. Spongy inside. Odor pungent. Taste slightly acrid. Spore-print whitish.
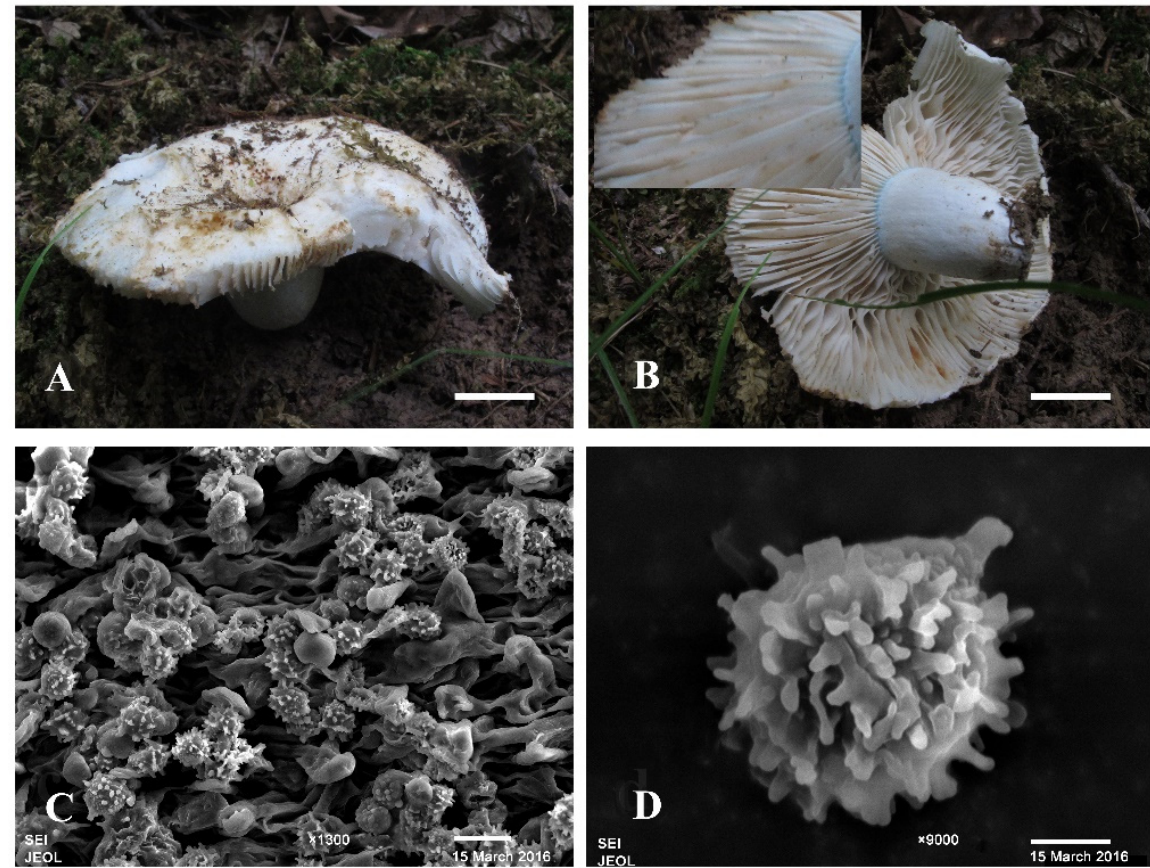

Figure 5. Basidiomata and microscopic structures of Russula callainomarginis (drawn from the holotype). (A,B) Basidiomata. (C,D) Basidiospores (SEM). Scale bars: $(\mathbf{A}, \mathbf{B})=1.3 \mathrm{~cm},(\mathbf{C}, \mathbf{D})=10 \mu \mathrm{m}$.
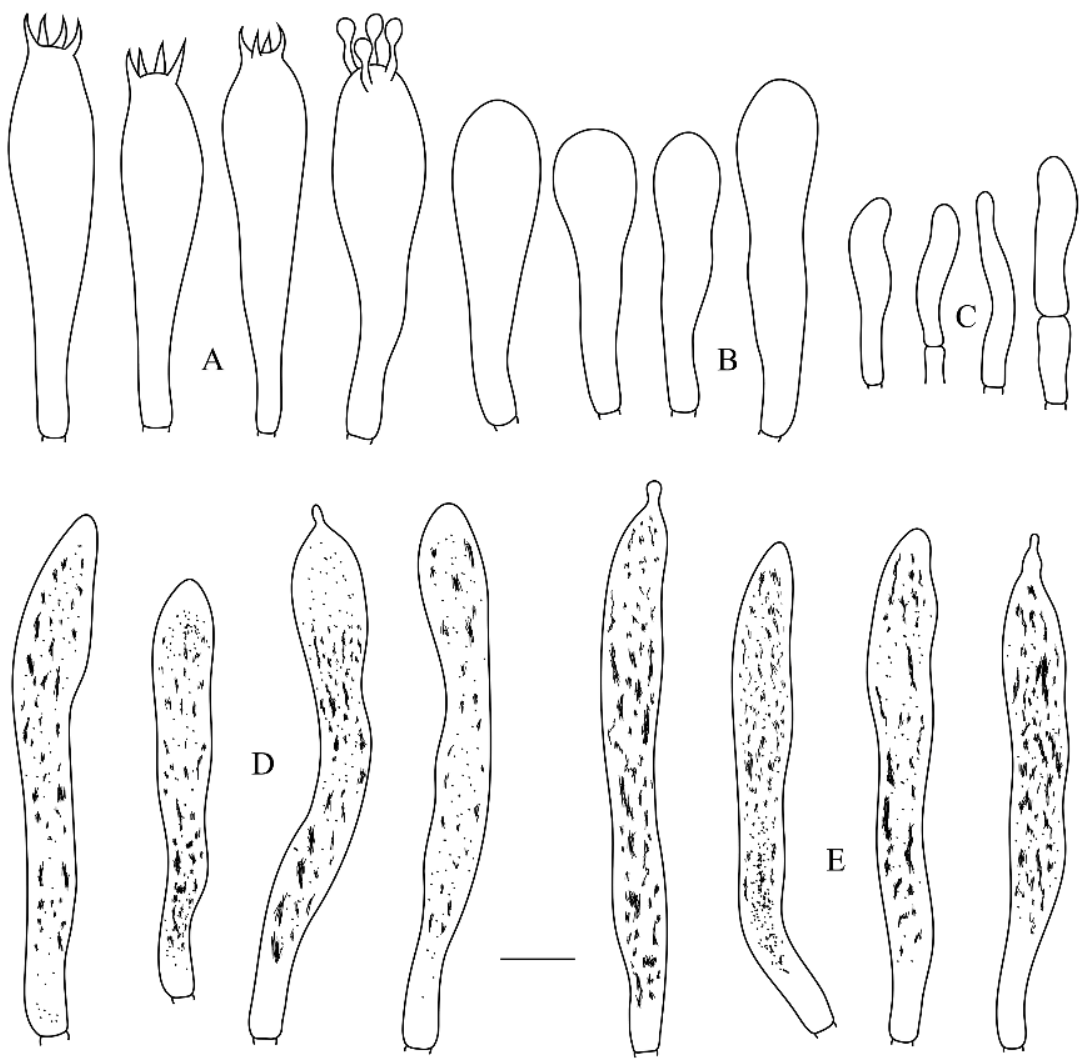

Figure 6. Russula callainomarginis (RITF2639). (A) Basidia. (B) Basidiola. (C) Marginal cells. (D) Hymenial cystidia on lamellae sides. (E) Hymenial cystidia on lamellae edges. Scale bar: $(\mathbf{A}-\mathbf{E})=10 \mu \mathrm{m}$. 

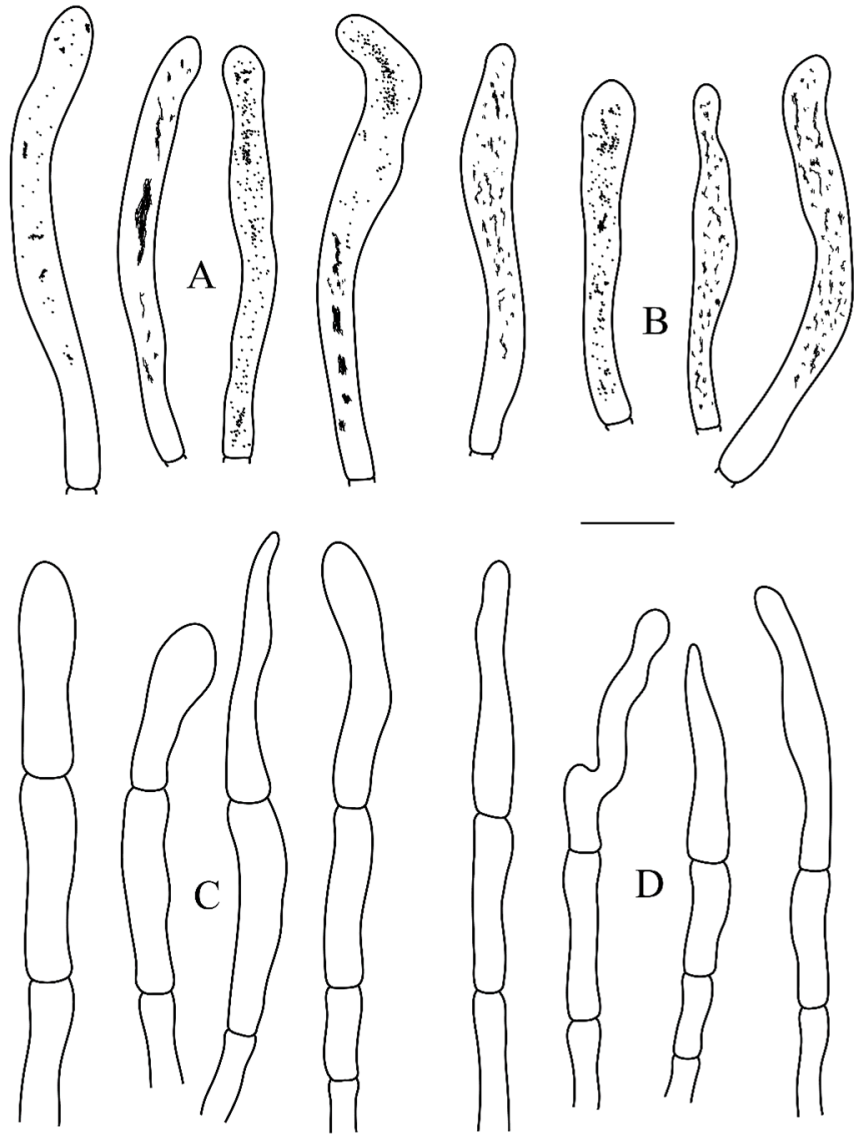

Figure 7. Russula callainomarginis (RITF2639). (A) Pileocystidia near the pileus margin. (B) Pileocystidia near the pileus center. (C) Hyphal terminations near the pileus margin. (D) Hyphal terminations near the pileus center. Scale bar: $(\mathbf{A}-\mathbf{D})=10 \mu \mathrm{m}$.

Basidiospores (6.4-)6.8-7.4-8.0(-8.5) × (5.2-)6.0-6.5-7.1(-7.5) $\mu \mathrm{m}, \mathrm{Q}=(1.0-) 1.07-1.13-$ $1.20(-1.31)$, globose to ellipsoid, ornamentation amyloid; warts bluntly conical to subcylindrical, up to $1 \mu \mathrm{m}$ high, isolated or connected with lines or ridges; suprahilar plage distinct, weakly amyloid. Basidia (40.0-)42.4-46.4-50.5(-52.0) × (10.2-)11.1-12.0-12.9(-13.6) $\mu \mathrm{m}$, mostly 4-spored, clavate or narrowly clavate; basidiola mainly clavate, ca. $8-13 \mu \mathrm{m}$ wide. Hymenial cystidia on lamellae sides moderately numerous, (57.7-)59.6-70.0-80.4(-93.0) $\times(7.6-) 8.0-8.8-9.5(-10.6) \mu \mathrm{m}$, clavate, fusiform or subcylindrical, apically often obtuse, occasionally with round or ellipsoid appendage, thin-walled; contents heteromorphous or granulose, turning dark grey in SV. Hymenial cystidia on lamellae edges similar to on lamellae sides in contents, often smaller, (55.3-)56.4-65.6-75.0(-88.0) × (6.8-)7.1-7.8-8.6(-9.3) $\mu \mathrm{m}$, fusiform or clavate, apically often obtuse, sometimes with round or ellipsoid appendage, thin-walled. Marginal cells (17.0-)18.0-20.6-23.2(-24.8) × (3.5-)3.9-4.5-5.0(-6.7) $\mu \mathrm{m}$, clavate, fusiform or subcylindrical, sometimes flexuous. Pilieipellis orthochromatic in Cresyl blue, not sharply delimited from the underlying spherocytes of the context, ca. 130-150 $\mu \mathrm{m}$ deep; vaguely divided in 80-100 $\mu \mathrm{m}$ deep subpellis, horizontally oriented, intricate, less gelatinized, dense hyphae, and ca. 50-70 $\mu \mathrm{m}$ deep suprapellis of strongly gelatinized, repent, loosely arranged hyphae forming a cutis, 3-7 $\mu \mathrm{m}$ wide. Hyphal terminations near the pileus margin rarely branched, sometimes flexuous, thin-walled, terminal cells (20.8-)22.5-28.0-33.6(-38.4) × (4.0-)4.6-5.7-6.8(-7.8) $\mu \mathrm{m}$, often clavate or narrowly lageniform, occasionally subcylindrical, apically obtuse or constricted; subterminal cells often longer, ca. 4-7 $\mu \mathrm{m}$ wide, typically unbranched. Hyphal terminations near the pileus center similar to those near the pileus margin, 19.7-23.5-27.2(-30.0) × (3.7-)3.9-4.8-5.8(-6.2) $\mu \mathrm{m}$, mainly subcylindrical and narrowly lageniform, occasionally clavate; subterminal cells often shorter, ca. 3-6 $\mu \mathrm{m}$ wide, typically unbranched. Pileocystidia near the pileus 
always one-celled, scattered, (29.3-)33.0-42.8-52.6(-58.3) × 4.4-5.6-6.7(-7.5) $\mu \mathrm{m}$, mainly clavate or subcylindrical, apically typically obtuse, thin-walled; contents granulose or crystalline, blackish in SV. Pileocystidia near the pileus center often shorter, always one-celled, scattered, (30.0-)33.1-38.6-44.0(-49.0) × 4.0-4.8-5.5(-6.0) $\mu \mathrm{m}$, mainly clavate or fusiform, apically typically obtuse, thin-walled; contents granulose or heteromorphous, turning dark grey in SV. Clamp connections absent in all tissues.

Habitat and distribution-single or scattered on ground in forest dominated by Fagaceae during July to September. Presently known only from central and south China.

Additional specimens (paratypes) examined-CHINA. Yunnan Province, Kunming, Heilongtan Forest Park, elev. 1950 m, 1 September 2012, Zhao 117 (HKAS 77470); Chuxiong, Lufeng County, Guangtong town, Xibaoqiao Village, on ground of Fagaceae, elev. 1883 m, 31 July 2015, Li150731-09 (LI); Shandong Province, Taian, Taohuayu, Caojiazhuang Village, elev. 297 m, 14 July 2016, Li160714-03 and Li160714-04 (LI); Guizhou Province, Guizhou, Qianlingshan Park, on ground of Fagaceae, elev. 1145 m, 10 September 2016, Li160910-20 (LI).

\section{Discussion}

The genus Russula has a cosmopolitan distribution from Arctic tundra to tropical forests and forms ectomycorrhizae with a diverse range of plants in deciduous, evergreen, broadleaf and coniferous forests, scrubland, and even meadows [5,35]. In China, 158 species had been confirmed by Li et al. 2014, with especially high species richness in Yunnan Province and the Greater and Lesser Khinggan Mountains [36], and new species are still being identified.

Our phylogeny generated a tree which is consistent with formerly published studies $[3,10,13]$. Both the macro- and micro-morphological characters (large basidiocarps, white or white to yellow ochre pileal surface when fresh, hard and rigid context, close to crowded lamellae with numerous lamellulae, short stipe, whitish spore-print and weakly amyloid plages), as well as the significant support for the phylogenetic placement of $R$. subbrevipes and $R$. callainomarginis $(\mathrm{BS}=100 \%, \mathrm{BPP}=1.00$ and $\mathrm{BS}=100 \%, \mathrm{BPP}=1.00$; Figure 1 ) together with $R$. delica, confirm that our new species belong to the subg. Brevipes.

Russula subbrevipes and R. callainomarginis share similar characters: unpeelable suprapellis, hard context, white spore-print, subglobose to ellipsoid basidiospores and amyloid ornamentation; however, $R$. subbrevipes differs from $R$. callainomarginis by producing a broadly infundibuliform pileus, yellow ochre pileal surface, tomentose stipe, fruity odor, partial connected warts, larger basidiospores, pleurocystidia with a cap appendage and never generating light turquoise lamellae margin.

Our phylogeny showed that $R$. brevipes was close to $R$. subbrevipes and $R$. callainomarginis (Figure 1). Morphologically, $R$. brevipes Peck resembles $R$. subbrevipes and $R$. callainomarginis by producing broadly medium-sized basidiomata, short stipe, close to crowded lamellae, whitish spore-print and amyloid ornamentation; however, $R$. brevipes differs from $R$. subbrevipes and $R$. callainomarginis in having a longer stipe $(30-80 \times 9-40 \mathrm{~mm})$ and warts $(0.7-2 \mu \mathrm{m})$, larger basidiospores $(8-11.3 \times 7.8-9.4 \mu \mathrm{m})$, subreticulate warts, indistinctive or mushroomy odor [37]. Moreover, R. brevipes has only been reported in coniferous forest to date and never generate a generating light turquoise lamellae margin and pleurocystidia with cap appendage [37-39].

Considering the broadly infundibuliform basidiomata, white spore-print, hard context and blue green zone on top of the stipe, $R$. delica and $R$. chloroides are similar to $R$. callainomarginis. However, $R$. delica differs from $R$. callainomarginis by the peppery or bitter taste, forking lamellae, longer basidiospores $(8-11.5 \times 6.5-8.7 \mu \mathrm{m})$; $R$. chloroides is distinguishable by yellowish to reddish pileal surface, larger basidiospores $(7-11 \times 6-8.7 \mu \mathrm{m})$, forking lamellae and peppery taste [6]. In addition, $R$. delica do not produce a light turquoise lamellae margin [6].

Russula leucocarpa, R. byssina and $R$. cremicolor fall into the $R$. subg. Brevipes and are also newly reported from China. However, these species were only found in coniferous forest, 
which is the remarkable difference. Morphologically, R. leucocarpa differs from $R$. subbrevipes by producing a smaller basidiocarp, forked lamellae and smaller basidiospores; $R$. byssina can be distinguished from $R$. subbrevipes by its smaller basidiocarp, peelable margin, shorter stipe and larger basidiospores; $R$. cremicolor differs from $R$. callainomarginis in its small basidomata, thick context and larger basidiospores [15].

Russula laevis Kälviäinen, Ruotsalainen and Taipale also resemble $R$. subbrevipes and $R$. callainomarginis by producing ochraceous cream to pale brownish pileus, short stipe, abundant lamellae, cream spore-print and amyloid ornamentation; However, the basidiospores are larger $((9.2-) 9.5-10-10.5(-11.3) \times(7.6-) 8-8.5-8.9(-9.6) \mu \mathrm{m})$, longer hymenial cystidia on lamellae $((72-) 79.5-86.8-94(-98) \times(7-) 7.5-8-8.5(-9.5))$, acrid taste, pileocystidia often with one or two central knobs and never generating a light turquoise lamellae margin [3].

For the time being, taxonomy and phylogeny of the Russula species in China has been relatively well-studied. However, many specimens are still unidentified in China, and comprehensive phylogeny of Russula at the genus level is lacking. Further studies based on broader sampling and more data are needed to clarify the fungi diversity and species affinities.

Author Contributions: Conceptualization, J.S.; data curation, J.S.; methodology, J.S. and B.C.; software, J.S. and B.C.; validation, J.S. and Q.C.; formal analysis, J.S. and H.L.; investigation, J.S. and S.W.; resources, J.S.; writing—original draft preparation, J.S. and B.C.; writing-review and editing, J.Z. and G.Y.; visualization, B.C.; supervision, J.L.; project administration, J.S.; funding acquisition, J.S. All authors have read and agreed to the published version of the manuscript.

Funding: This research was supported by the National Natural Science Foundation of China (Project No. 31900016).

Institutional Review Board Statement: Not applicable for studies not involving humans or animals. Informed Consent Statement: Not applicable for studies not involving humans.

Data Availability Statement: Publicly available datasets were analyzed in this study. These data can be found here: https:/ / www.ncbi.nlm.nih.gov/, accessed on 27 January 2016; https:/ /www.mycoba nk.org/page/Simple\%20names\%20search, accessed on 27 January 2016; http:/ / purl.org/phylo/treeb ase / phylows / study /TB2:S23830?x-access-code=71a348fee3ccd4c31d60764d310ebf3andformat=html, accessed on 27 January 2016.

Conflicts of Interest: The authors declare no conflict of interest.

\section{References}

1. Persoon, C.H. Observations Mycologicae, Seu, Descriptiones Tam Novorum Quan Notabilium Fungorum; Apud Petrum P. Wolf: Lipsiae, Germany, 1796.

2. Kirk, P.M.; Cannon, P.F.; David, J.C.; Minter, D.W.; Stalpers, J.A. Ainsworth and Bisby's Dictionary of the Fungi, 10th ed.; CAB International Press: Wallingford, UK, 2008.

3. Adamčík, S.; Looney, B.; Caboň, M.; Jančovičová, S.; Adamčíková, K.; Avis, P.G.; Barajas, M.; Bhatt, R.P.; Corrales, A.; Das, K.; et al. The quest for a globally comprehensible Russula language. Fungal Divers. 2019, 99, 369-449. [CrossRef]

4. Buyck, B.; Hofstetter, V.; Eberhardt, U.; Verbeken, A.; Kauff, F. Walking the thin line between Russula and Lactarius: The dilemma of Russula subsect. Ochricompactae. Fungal Divers. 2008, 8, 15-40.

5. Looney, B.P.; Meidl, P.; Piatek, M.J.; Miettinen, O.; Martin, F.M.; Matheny, P.B.; Labbé, J.L. Russulaceae: A new genomic dataset to study ecosystem function and evolutionary diversification of ectomycorrhizal fungi with their tree associates. New Phytol. 2018, 218, 54-65. [CrossRef] [PubMed]

6. Romagnesi, H. Statuts et noms nouveaux pour les taxa infragénériques dans le genre Russula. Doc. Mycol. 1987, 18, 39-40.

7. Singer, R. The Agaricales in Modern Taxonmy, 4th ed.; Koeltz Scientific Books: Königstein im Taunus, Germany, 1986.

8. Bon, M. Clé onographique des russules d'Europe. Doc. Mycol. 1988, 18, 1-120.

9. Sarnari, M. Monografia Illustrate de Genere Russula in Europa. Tomo Primo; AMB, Centro Studi Micologici: Trento, Italy, 1998.

10. Buyck, B.; Zoller, S.; Hofstetter, V. Walking the thin lin ten years later: The dilemma of above- versus below-ground features to support phylogenies in the Russulaceae (Basidiomycota). Fungal Divers. 2018, 89, 267-292. [CrossRef]

11. Buyck, B.; Wang, X.H.; Adamčíková, K.; Caboň, M.; Jančovičová, S.; Hofstetter, V.; Adamčík, S. One step closer to unravelling the origin of Russula: Subgenus Glutinosae subg. nov. Mycosphere 2020, 11, 285-304. [CrossRef] 
12. Hongsanan, S.; Hyde, K.D.; Bahkali, A.H.; Camporesi, E.; Chomnunti, P.; Ekanayaka, H.; Gomes, A.A.M.; Hofstetter, V.; Jones, E.B.G.; Pinho, D.B.; et al. Fungal Biodiversity Profiles 11-20. Cryptog. Mycol. 2015, 36, 355-380. [CrossRef]

13. Eberhardt, U. Molecular kinship analyses of the agaricoid Russulaceae: Correspondence with mycorrhizal anatomy and sporocarp features in the genus Russula. Mycol. Prog. 2002, 1, 201-223. [CrossRef]

14. Miller, S.L.; Buyck, B. Molecular phylogeny of the genus Russula in Europe with a comparison of modern infrageneric classifications. Mycol. Res. 2002, 106, 259-276. [CrossRef]

15. Li, G.J.; Deng, C.Y.; Shi, L.Y.; Wang, J.; Meng, Q.F.; Li, S.M. Three new species of Russula subsect. Lactarioideae from China. Mycosystema 2020, 39, 618-636.

16. Jiang, X.M.; Li, Y.K.; Liang, J.F.; Wu, J.R. Russula brunneovinacea sp. nov., from north-eastern China. Mycotaxon 2018, $132,789-797$. [CrossRef]

17. Chen, B.; Song, J.; Chen, Y.1.; Zhang, J.H.; Liang, J.F. Morphological and phylogenetic evidence for two new species of Russula subg. Heterophyllidia from Guangdong Province of China. MycoKeys 2021, 82, 139-157.

18. Li, G.J.; Li, S.M.; Buyck, B.; Zhao, S.Y.; Xie, X.J.; Shi, L.Y.; Deng, C.Y.; Meng, Q.F.; Sun, Q.B.; Yan, J.Q.; et al. Three new Russula species in sect. Ingratae (Russulales, Basidiomycota) from southern China. MycoKeys 2021, 84, 103-139. [CrossRef] [PubMed]

19. Song, Y.; Xie, X.C.; Buyck, B. Two novel species of subgenus Russula crown clade (Russulales, Basidiomycota) from China. Eur. J. Taxon. 2021, 775, 15-33. [CrossRef]

20. Vellinga, E.C.; Noordeloos, M.E. Glossary. In Flora Agaricina Neerlandica 5; Noordeloos, M.E., Kuyper, T.W., Vellinga, E.C., Eds.; A.A. Balkema Publishers: Rotterdam, The Netherlands, 1988; pp. 6-11.

21. Kornerup, A.; Wanscher, J.H. Taschenlexikon der Farben, 3rd ed.; Muster-Schmidt Verlag: Göttingen, Germany, 1981.

22. Buyck, B. Valeur taxonomique du bleu de crésyl pour le genra Russula. Bull. De La Société Mycol. Fr. 1989, 120, 1-6.

23. Zhou, L.L.; Liang, J.F. An improved protocol for extraction of DNA from macrofungi. Guangdong Fore. Sci. Tech. 2011, $27,13-16$.

24. White, T.J.; Bruns, T.; Lee, S.; Taylor, J. Amplification and Direct Sequencing of Fungal Ribosomal RNA Genes for Phylogenetics. PCR Protocols: A Guide to Methods and Applications; Academic Press: New York, NY, USA, 1990; Volume 18, pp. $315-322$.

25. Vilgalys, R.; Hester, M. Rapid genetic identification and mapping enzymatically amplified ribosomal DNA from several Cryptococcus species. J. Bacteriol. 1990, 172, 4238-4246. [CrossRef]

26. Liu, Y.J.; Hall, B.D. Body plan evolution of ascomycetes, as inferred from an RNA polymerase II, phylogeny. Proc. Natl. Acad. Sci. USA 2004, 101, 4507-4512. [CrossRef]

27. Matheny, P.B. Improving phylogenetic inference of mushrooms with RPB1 and RPB2 nucleotide sequences (Inocybe; Agaricales). Mol. Phyl. Evol. 2005, 35, 1-20. [CrossRef]

28. Liang, J.F.; Xu, J.; Yang, Z.L. Divergence, dispersal and recombination in Lepiota cristata from China. Fungal Divers. 2009, 38, 105-124.

29. Nilsson, R.H.; Tedersoo, L.; Abarenkov, K.; Ryberg, M.; Kristiansson, E.; Hartmann, M.; Schoch, C.L.; Nylander, J.A.A.; Bergsten, J.; Porter, T.M.; et al. Five simple guidelines for establishing basic authenticity and reliability of newly generated fungal ITS sequences. Mycokeys 2012, 4, 37-63. [CrossRef]

30. Katoh, K.; Toh, H. Recent developments in the MAFFT multiple sequence alignment program. Brief Bioinform. 2008, 9, 286-298. [CrossRef] [PubMed]

31. Hall, T.A. BioEdit: A user-friendly biological sequence alignment editor and analysis program for Windows 95/98/NT. Nucleic Acids Symp. Ser. 1999, 41, 95-98.

32. Swofford, D.L. PAUP*: Phylogenetic Analysis Using Parsimony (*and Other Methods). Version 4.0b10; Sinauer Associates: Sunderland, MA, USA, 2002.

33. Posada, D.; Crandall, K.A. Model test: Testing the model of DNA substitution. Bioinformatics 1998, 14, 817-818. [CrossRef] [PubMed]

34. Nylander, J.A.A. MrModeltest 2.3. Computer Program and Documentation Distributed by the Author; Evolutionary Biology Centre, Uppsala University: Uppsala, Sweden, 2004.

35. Knudsen, H.; Borgen, T. Russulaceae in Greenland. Arctic and Alpine Mycology 1; University of Washington Press: Seattle, WA, USA, 1982.

36. Li, G.J.; Li, S.F.; Zhao, D.; Wen, H.A. Recent research progress of Russula (Russulales, Agaricomycetes): A review. Mycosystema 2015, 34, 821-848.

37. Roberts, C. Russulas of Southern Vancouver Island Coastal Forests. Doctoral Dissertation, University of Victoria, Victoria, BC, Canada, 2007.

38. Shaffer, R.L. Notes on the subsection Crassotunicatinae and other species of Russula. Lloydia 1970, 33, 49-96.

39. Buyck, B.; Adamčík, S. Type studies in Russula subsection Lactarioideae from North America and a tentative key to North American species. Cryptogam. Mycol. 2013, 34, 259-279. [CrossRef] 